\title{
Mixed neuroendocrine nonneuroendocrine neoplasms of the pancreas: a case report and literature review of pancreatic mixed neuroendocrine nonneuroendocrine neoplasm
}

\author{
Fei Wang ${ }^{1,2,3,4,5 \#}$, Xin Lou ${ }^{1,2,3,4,5 \#}$, Yi Qin ${ }^{1,2,3,4,5}$, Xiaowu Xu ${ }^{1,2,3,4,5}$, Xianjun Yu ${ }^{1,2,3,4,5}$, Dan Huang ${ }^{6}$, \\ Shunrong Ji ${ }^{1,2,3,4,5}$
}

${ }^{1}$ Center for Neuroendocrine Tumors, Fudan University Shanghai Cancer Center, Shanghai, China; ${ }^{2}$ Department of Pancreatic Surgery, Fudan University Shanghai Cancer Center, Shanghai, China; ${ }^{3}$ Department of Oncology, Shanghai Medical College, Fudan University, Shanghai, China; ${ }^{4}$ Shanghai Pancreatic Cancer Institute, Shanghai, China; ${ }^{5}$ Pancreatic Cancer Institute, Fudan University, Shanghai, China; ${ }^{6}$ Department of Pathology, Fudan University Shanghai Cancer Center, Shanghai, China

"These authors contributed equally to this work and should be considered as co-first authors.

Correspondence to: Dan Huang. Department of Pathology, Fudan University Shanghai Cancer Center, Shanghai 200032, China.

Email: danhuang@shca.org.cn; Shunrong Ji. Center for Neuroendocrine Tumors, Fudan University Shanghai Cancer Center, Shanghai 200032,

China. Email: jishunrong@fudanpci.org.

\begin{abstract}
A 36-year-old woman underwent left lateral hepatic lobectomy and tumor enucleation of the pancreatic head due to the diagnosis of mixed neuroendocrine nonneuroendocrine neoplasms (MiNENs). Her past medical history revealed an ill-defined mass in the pancreatic uncinate (approximately $1 \mathrm{~cm}$ in size) and liver nodules in segment II, suggesting a pancreatic neuroendocrine tumor (pNET) and liver metastases. After the operation, pathological examination showed that the lesion in the pancreas was MiNEN. The neuroendocrine component consisted of grade 1 (G1) NETs, and the nonneuroendocrine part was welldifferentiated adenocarcinoma. Additionally, in hepatic lesions, the type of pancreaticobiliary duct in moderately differentiated adenocarcinoma indicated the source of tumor cells. We present the following case in accordance with the CARE reporting checklist. MiNENs of the pancreas are extremely rare and heterogeneous malignancies. Owing to the rarity of pancreatic MiNEN, no structured guidelines on preoperative diagnostics, therapy and follow-up exist thus far. Thus, problems in differential diagnosis and appropriate therapy may arise when treating suspected MiNEN patients. We performed a rigorous search on pancreatic MiNEN via PubMed and Web of Science. In total, 167 studies were identified, and 28 cases in 15 studies about pancreatic MiNEN were assessed. All the available information was listed and fully discussed.
\end{abstract}

Keywords: Mixed neuroendocrine nonneuroendocrine neoplasm (MiNEN); pancreas; pancreatic mixed neuroendocrine nonneuroendocrine neoplasm; case report

Submitted Aug 24, 2021. Accepted for publication Nov 08, 2021.

doi: 10.21037 /gs-21-564

View this article at: https://dx.doi.org/10.21037/gs-21-564

\section{Introduction}

Mixed neuroendocrine nonneuroendocrine neoplasm (MiNEN) in the pancreas is an extremely rare and heterogeneous malignancy. In 1987, the first classification of mixed neoplasms with neuroendocrine and nonneuroendocrine components was proposed by Lewin (1). Thirteen years later, Capella et al. (2), attempted to standardize the terminology of digestive mixed neoplasms

^ ORCID: 0000-0002-2517-079X. 
and provide a prognostic classification. In the same year, the World Health Organization (WHO) decided to introduce the 'mixed exocrine-endocrine tumor (MEEC)' to define neoplasms consisting of a nonneuroendocrine (exocrine) component and a neuroendocrine component, and the $30 \%$ cutoff value for each component was also defined. In 2010, 'MEEC' was replaced by 'mixed adeno-neuroendocrine carcinomas (MANECs)' by the WHO because of the wider real spectrum of mixed neoplasms in the digestive tract, while the cutoff remained. MANEC failed to adequately convey the morphological and biological heterogeneity of digestive mixed neoplasms, so the WHO reclassified MANEC to MiNEN in $2017(3,4)$.

MiNENs are defined as mixed neoplasms with both neuroendocrine and nonneuroendocrine components, in which either component represents at least $30 \%$ of the lesion. MiNEN are generally thought to have an aggressive nature and to be associated with poor prognosis (5). While MiNEN have been found in several organs (e.g., stomach, intestines, pancreas, biliary tract, appendix, and cervix), the pancreas and gastrointestinal tract are the most common places they are usually found $(4,6)$. In this study, we report a patient with small $(\sim 1 \mathrm{~cm})$ primary pancreatic MiNEN with liver metastasis constituted by adenocarcinoma. Informed consent was obtained from the patient. A review of the English literature about pancreatic MiNEN was performed via PubMed (MEDLINE) and Web of Science with a rigorous search strategy (see Appendix 1). All abstracts, studies, and citations obtained were carefully reviewed by two investigators. In total, 167 articles were identified, and 28 cases in 15 studies about pancreatic MiNEN were assessed, listed, and fully discussed. We present the following case in accordance with the CARE reporting checklist (available at https://dx.doi.org/10.21037/gs-21-564).

\section{Case presentation}

A 36-year-old woman was admitted to a local hospital due to an ill-defined mass in the pancreatic uncinate (approximately $1 \mathrm{~cm}$ in size) and liver nodules in segment II during a regular medical check-up. Further laboratory examination showed a slightly elevated serum carbohydrate antigen 19-9 (CA19-9) level (41.30 U/mL; normal value $<37.0 \mathrm{U} / \mathrm{mL}$ ) but normal AFP and CEA level. She did not present with abdominal pain and distension, nausea, vomiting, diarrhea, or hypoglycemia.

For further treatment, she was referred to our hospital. Enhanced computed tomography (CT) of the abdomen revealed a markedly enhanced lesion $(1.0 \mathrm{~cm} \times 0.9 \mathrm{~cm})$ in the pancreatic head (Figure 1A) and a low-density lesion with slight enhancement on the periphery in the liver (Figure 1B). The patient underwent abdominal magnetic resonance imaging (MRI) for further evaluation. The MRI revealed a focal nodule with obvious enhancement in the pancreatic head (Figure 1C). In the left hepatic lobe, an irregular nodule was found, approximately $17 \mathrm{~mm}$, with edge intensification in the arterial phase, hyposignal in the T1-weighted image (T1W1) and a relatively low apparent diffusion coefficient (ADC) value (Figure 1D). According to $\mathrm{CT}$ and MR examination, the lesion of the pancreatic head was considered a neuroendocrine tumor (NET), while the nature of the liver was unclear. The majority of pNETs express somatostatin receptors (SSTRs) on cell surfaces, especially in well-differentiated tumors (G1 or G2) with a low Ki-67 index; thus, gallium-68 TATE positron emission tomography/CT (68-Ga-TATE-PET/CT) was considered to assess SSTR expression (7). Then, 68-Ga-TATE-PET/ CT was performed and it revealed increased focal TATE uptake in the head of the pancreas (SUVmax $=8.5$ ) and no increased TATE uptake in the slightly hypodense lesion of the left liver (Figure 2), which confirmed our previous diagnosis of the pancreatic lesion. After a multidisciplinary team (MDT) meeting, liver biopsy was suggested to determine the nature of the lesion. The pathology turned out to be adenocarcinoma.

As previously mentioned, NETs (G1 and G2) normally express SSTR on the cell surface, while the SSTR decreases when tumors become less differentiated and more aggressive. With the decline in SSTR expression, cell glucose utilization increases (8). In ${ }^{18} \mathrm{~F}$-fluorodeoxyglucose PET/CT (18-F-FDG-PET/CT) images, FDG, as a glucose analog, could visualize glucose metabolic activity. Since the primary lesion was unknown, 18-F-FDG PET/CT was also performed, which showed elevated FDG uptake only in the lesion of the left liver lobe (SUVmax =6.2). Gastroscopy and colonoscopy, as well as gynecological examinations, were then performed to look for possible primary lesions. However, no abnormalities were found.

After another MDT meeting and communication with the patient, we performed a laparoscopic exploration on 8th May 2021. Pancreatic head nodules and hepatic nodules in segment II were found. No peritoneal metastasis was observed. Thus, the patient underwent left lateral hepatic lobectomy and tumor enucleation of the pancreatic head via laparoscopy.

Postoperative pathological examination showed 

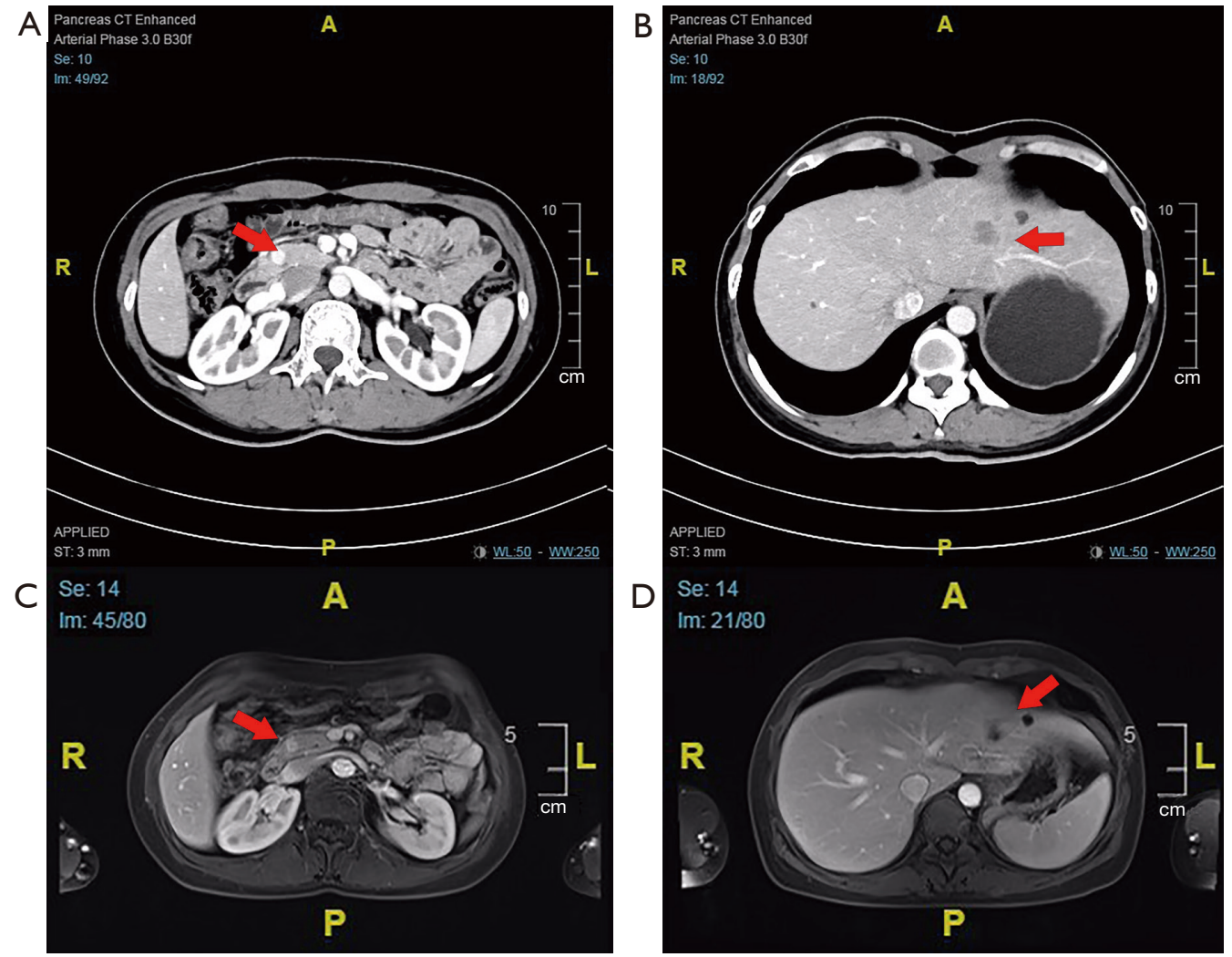

Figure $1 \mathrm{CT}$ scan and MRI. (A) The enhanced CT scan reveals a markedly enhanced lesion in the pancreatic head $(1.0 \mathrm{~cm} \times 0.9 \mathrm{~cm})$. (B) A low-density lesion with slightly enhanced on the periphery of liver in the enhanced CT imaging. (C) MRI revealed a focal nodule with obvious enhancement in the pancreatic head. (D) A nodule (approximately $17 \mathrm{~mm}$ ) with edge intensifying in arterial phase was found in in the left hepatic lobe on MRI. The arrows indicate the primary tumor in the head of pancreas and the metastatic liver lesion, respectively. CT, computed tomography; MRI, magnetic resonance imaging.

that the hepatic lesions were moderately differentiated adenocarcinoma with a type of pancreaticobiliary duct, while the pancreatic lesion were MiNENs (Figure 3). The neuroendocrine component consisted of grade 1 (G1) NETs, and the nonneuroendocrine part was a well-differentiated adenocarcinoma. According to hematoxylin and eosin (HE) staining, the composition of adenocarcinoma accounted for approximately $30 \%$. The neuroendocrine part was immunohistochemically positive for cytokeratin-multi-AE1-AE3 (AE1/AE3), synaptophysin, chromogranin $\mathrm{A}$, insulinoma-associated protein 1 and SSTR type 2 and negative for SSTR type 3. In addition, Ki67 index was approximately $2 \%$. These features indicated NET, G1. Moreover, the hepatic lesions showed moderately differentiated adenocarcinoma with a type of pancreaticobiliary duct. Immunohistochemical results were positive for cytokeratin 7 and cytokeratin 19, but negative for anti-CDX2 antibody (CDX2-88) and special AT-rich sequence binding protein 2 (SATB2). Due to the presence of liver metastasis, chemotherapy was given. Clinical course of the patient was shown in Figure 4.

All procedures performed in studies involving human participants were in accordance with the ethical standards of the institutional and/or national research committee(s) and with the Helsinki Declaration (as revised in 2013). Written informed consent was obtained from the patient for publication of this case report and accompanying images. A copy of the written consent is available for review by the editorial office of this journal.

\section{Discussion}

MiNENs of the pancreas are extremely rare and heterogeneous malignancies. Based on previous studies, 

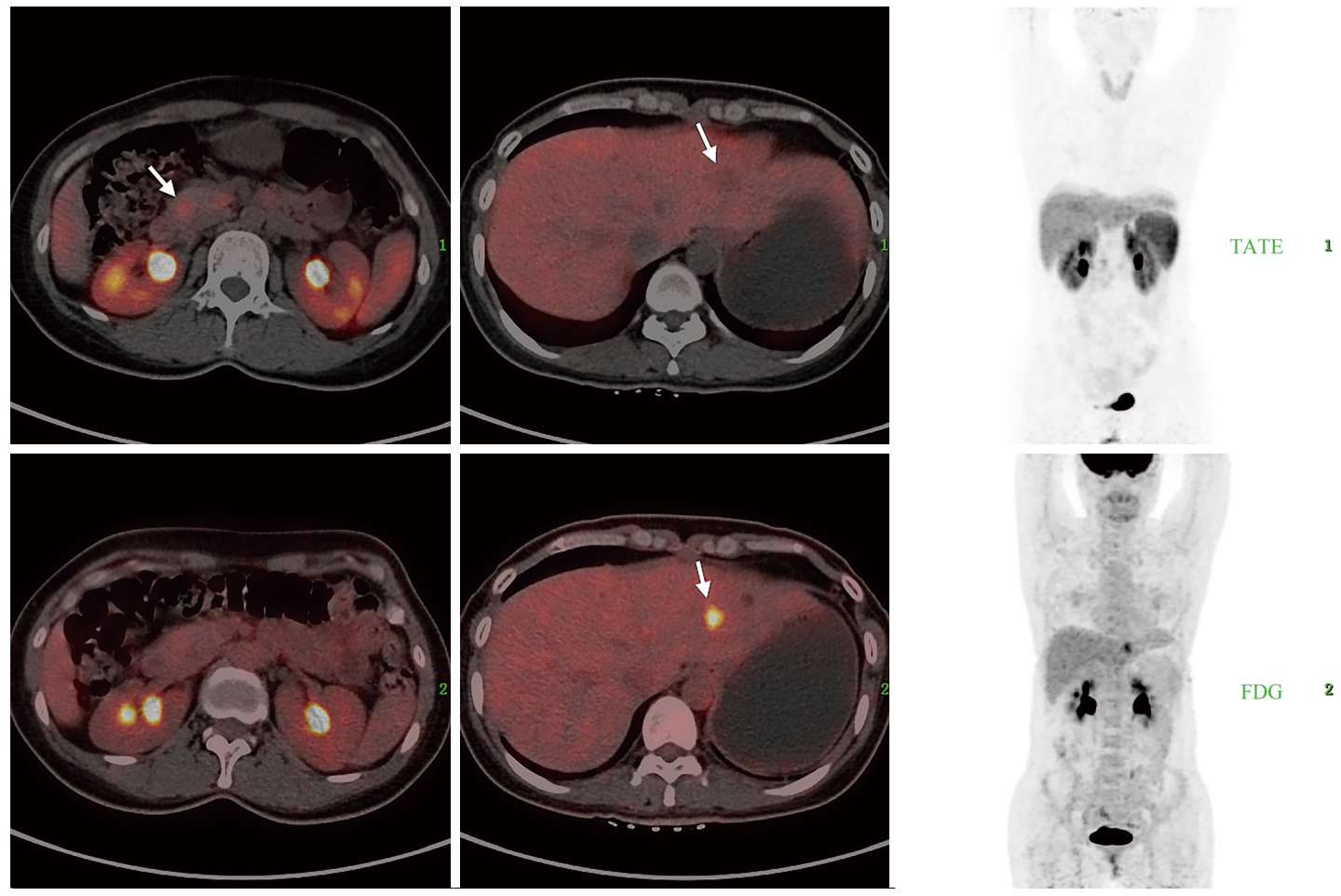

Figure 2 PET/CT scans using 68-Ga-TATE and ${ }^{18}$ F-FDG. The arrows indicate the primary tumor in the head of pancreas and the metastatic liver lesion, respectively. PET/CT, positron emission tomography/computed tomography.
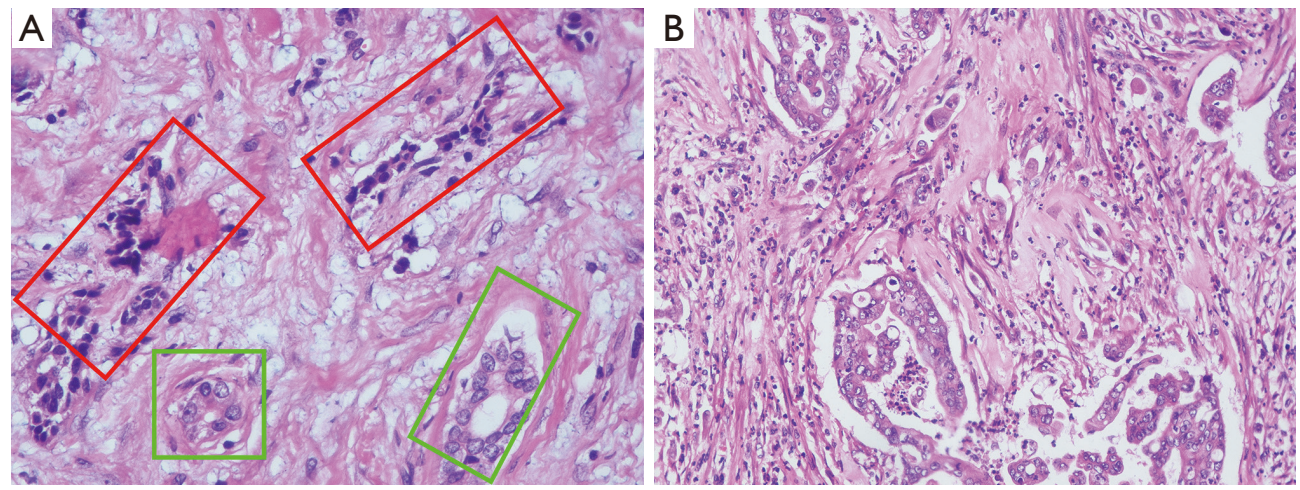

Figure 3 Histopathological findings. (A) The tissue of pancreatic lesion stained with HE (field of view: 10×40). The neuroendocrine component consisting of neuroendocrine tumor and nonneuroendocrine part consisting of adenocarcinoma are in the red-framed and light green-framed areas, respectively. (B) The tissue of hepatic lesion stained with HE (field of view: 10×20). HE, hematoxylin and eosin.

albeit limited and conflicting, MiNEN is an aggressive entity with a high-grade neuroendocrine component in most cases and is associated with poor survival outcomes similar to those of pure neuroendocrine carcinomas (NECs) $(9,10)$. However, in our case, the neuroendocrine component was G1 NETs, which are rarer. Due to the rarity of this diagnosis, evidence from the literature on MiNENs is almost exclusively derived from case reports and small retrospective series. With inadequate published data, the epidemiology, prognosis, and best therapeutic management of patients with MiNEN remain unknown.

We performed a rigorous search on pancreatic MiNEN cases via PubMed and Web of Science (last search on 14th August 2021). In total, 167 studies were identified 


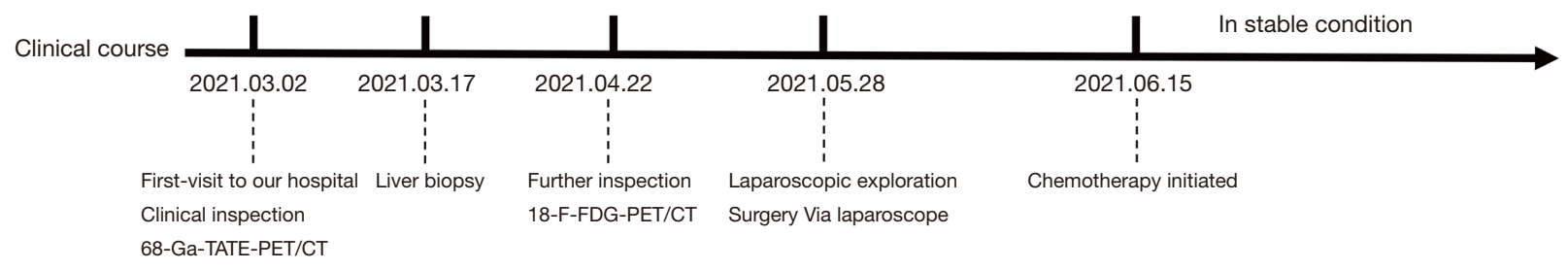

Figure 4 Clinical course of the patient. PET/CT, positron emission tomography/computed tomography.

(66 from PubMed and 101 from Web of Science). After removing duplications, 101 articles underwent title and abstract assessment, of which 34 articles were admitted to the full text screen. An additional related study was found and enrolled while screening the reference lists. Eventually, 28 cases in 15 studies about pancreatic MiNEN were assessed (Table 1). The studies yielded, patient age which ranged from 30 to 82 years (average age was 61.2 years) and the male to female ratio was $3: 1$. According to the table, MiNEN were located variably in all parts of the pancreas (10 in the head, 5 in the body, 3 in the tail, 7 in the ampulla of Vater and 3 in the uncinate process of the pancreas). Among them, 11 patients presented with metastatic lesions, and 8 of them had liver metastases. While most patients resorted to surgery, unresectable patients received chemotherapy as the main treatment.

Pancreatic MiNEN could arise in any part of the pancreas; for the histogenesis of pancreatic MiNEN, various hypotheses have been proposed. Some studies suggested that associated neuroendocrine and nonneuroendocrine neoplasms in the pancreas may arise from totipotent pancreatic stem cells in the pancreatic duct and islets, while other reporters assumed that dysfunction of numerous tumor suppressor genes resulted in different types of malignancy of MiNEN (13,25-27). In 2014, Scardoni et al. (28), suggested a monoclonal origin of the two MiNEN (MANCE) components of the gastrointestinal tract based on next-generation sequencing. In a recent study by Schiavo Lena et al. (12), they reported a case of pancreatic mixed intraductal papillary mucinous neoplasm-NET and confirmed the true mixed nature of both components based on the same molecular alterations (KRAS, GNAS, and $C D K N 2 A$ mutations, and the amplification of the CCND1 gene).

In MiNEN, acinar adenocarcinoma as a nonneuroendocrine component has been reported more frequently in the literature, while ductal adenocarcinoma as a component has rarely been reported. In most digestive MiNENs, the neuroendocrine component is represented by NEC, and accordingly, the patient's outcome is ominous (29,30). In pancreatic MiNENs, mixed ductal-NEC (the main type of adeno-NEC) and mixed acinar-NEC are two main entities (29). A previous study reported that digestive NECs and adenocarcinomas share similar genetic mutation landscapes, suggesting a monoclonal origin of the two MiNEN components (28). Interestingly, this MiNEN case we reported consisted of well-differentiated NETs and adenocarcinoma, but the evolutionary relationship between NETs and adenocarcinoma is still unclear, and whether NETs can transcend NECs to evolve into adenomas also demands further research. Therefore, the next-generation sequencing data for this case are being processed, and our team is committed to solving these problems.

For pancreatic adeno-MiNEN, Yang et al. (5), performed a Chinese single-center study on pancreatic neuroendocrine neoplasms (pNEN) over a study period of 10 years (20022012) and showed a frequency of 5.5\% (6 of 110) adenoMiNEN out of all pNEN patients resected at the center. In the study of Nießen et al. (11), found that the occurrence of adeno-MiNEN was $0.2 \%$ (8 of 3,580) of all pancreatic resections for pancreatic tumors and $1.4 \%$ (8 of 570) of all resected pNEN over a study period of 19 years (2001-2019). For pancreatic acinar-MiNEN, Pokrzywa et al. (30), found that 515 of 57,804 patients resected for pancreatic malignancy had pancreatic acinar-MiNEN via American National Cancer Base, with an incidence of $0.9 \%$ over ten years (2004-2014). Similarly, in the cohort of Nießen et al. (11), the frequency of acinar-MiNEN was $0.8 \%$ (5 of 570 ) of all resected pNENs and $0.14 \%$ (5 of 3,580 patients) of pancreatic resections for pancreatic tumors from 2001 to 2019.

Pancreatic MiNEN usually present nonspecific symptoms due to local tumor growth and/or metastatic dissemination, leading to difficulty in diagnosis. HE-stained sections served for the morphological identification of both neuroendocrine and nonneuroendocrine components. Immunohistochemistry is mandatory to test the nature of the neuroendocrine component, which is also recommended to evaluate the subtype of the nonneuroendocrine 


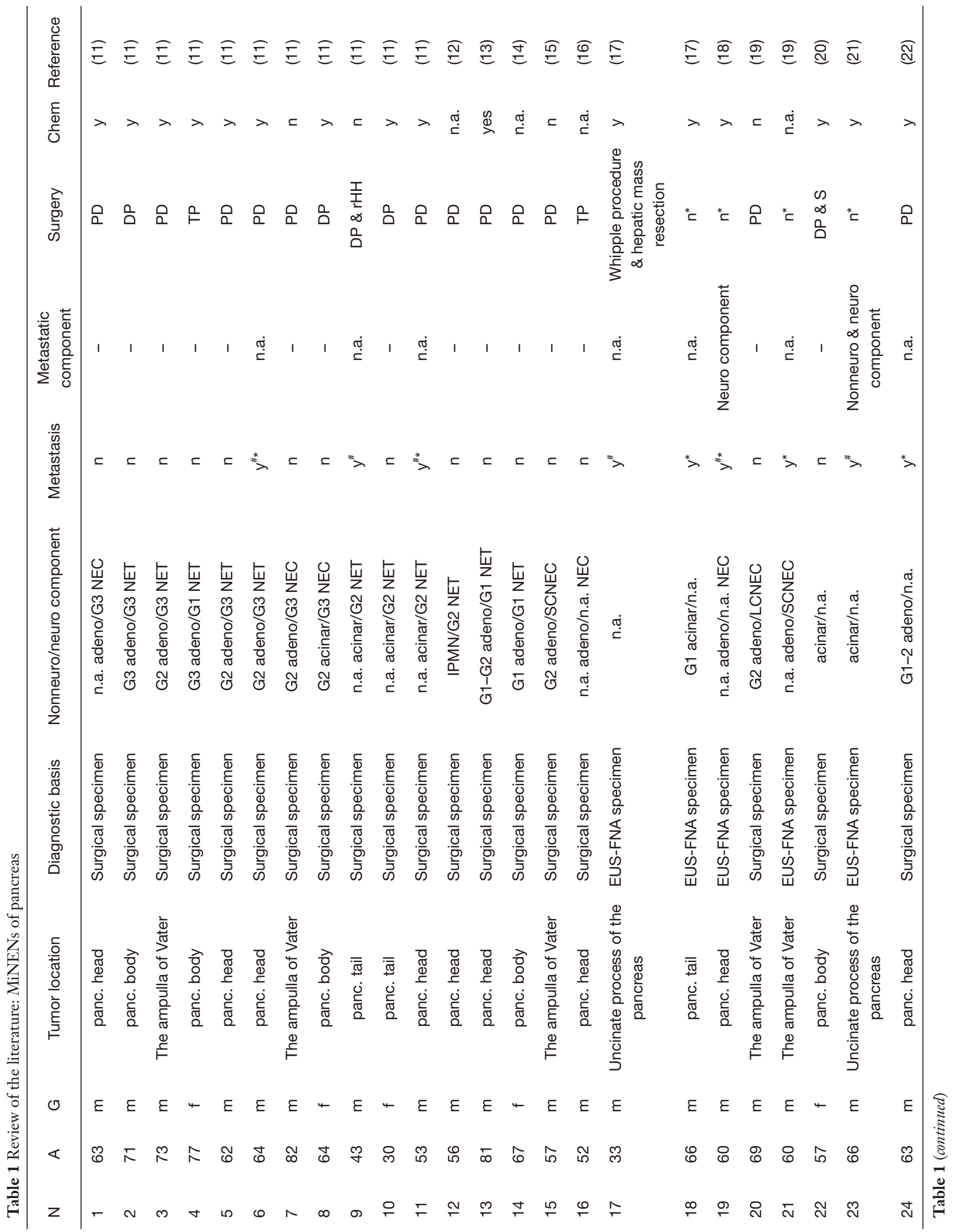




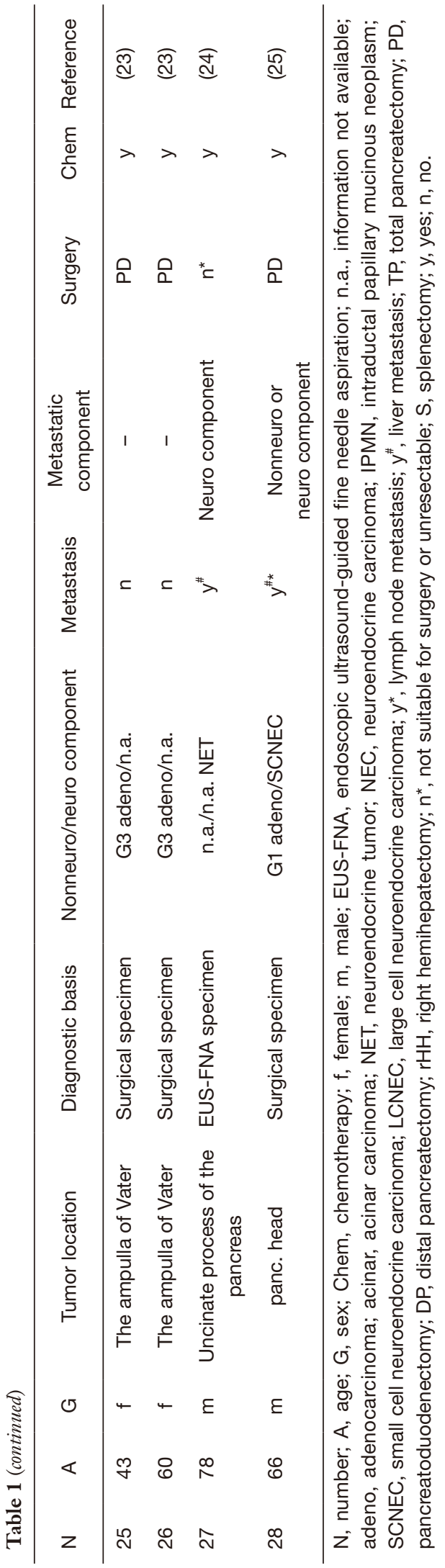

part in MiNEN. Histopathological examination with immunohistochemistry of surgically resected specimens is the gold standard to confirm MiNEN; most patients were previously suspected to have pancreatic tumors or pNEN, and the final diagnosis of MiNEN was made based on the pathological evaluation of surgical specimens $(11-13,15,16,19,20,22,23,25)$. Preoperative diagnosis was rarely established, and only a few cases reported had been diagnosed through preoperative biopsy with endoscopic ultrasound-guided fine needle aspiration (EUS-FNA) specimens (17-19,21,24). Recently, EUS-FNA, a minimally invasive technique for histological diagnoses, has been widely used to diagnose pancreatic tumors, and several reports have used it for preoperative diagnosis in pancreatic MiNEN. However, it is not used in all MiNEN cases and should be carefully considered before use because it has high negative predictive value and fails to access the whole tumor to diagnose MiNEN (13).

The management and treatment of pancreatic MiNEN patients are without uniform standards. Usually, patients undergo surgery (normally R0 resection), and adjuvant chemotherapy is probably arranged after surgery (13). However, it is unclear whether to focus on both components or the major component. La Rosa et al. (4), suggested that treatment for MiNEN should be based on the dominant component because the clinical outcome is usually determined by the more aggressive cell type of such mixed tumor. This opinion was shared by most investigators and clinicians.

For prognosis in adeno-MiNEN, Yang et al. (5), reported on 6 adeno-MiNEN patients with a median survival of 15.3 months. In the literature with ten surgically resected gastroenteropancreatic adeno-MiNEN patients, the median follow-up duration was 15 months (range, 3-51 months) (31). According to the data from Nießen et al. (11), among 13 patients with MiNEN, the 5-year overall survival (OS) rate in all MiNEN was $40 \%$, and the 5 -year survival rate was $58 \%$ in adeno-MiNEN compared to matched pNEN (48\%) and ductal adenocarcinomas (36\%). For acinar-MiNEN, Kim et al. (32), compared 20 acinar cell carcinomas (ACCs) and 13 acinar MiNEN to a cohort of 269 welldifferentiated pancreatic neuroendocrine tumors (pNETs). The study indicated that patients with acinar MiNEN had worse overall $(\mathrm{P}<0.001)$ recurrence-free survival $(\mathrm{P}<0.001)$ than those with pNETs, with a 5 -year OS rate of $46.7 \%$. Similarly, in the study of Pokrzywa et al. (30), the median OS and 5-year OS rates were 26.8 months and $37 \%$, respectively, based on the evaluation of 515 acinar MiNEN 
patients between 2004 and 2014. In the cohort of Nießen et al. (11), median and 5-year OS were lower for acinarMiNEN than in previous literature, 17 months and 20\%, respectively.

In conclusion, we report a new case of MiNEN with liver metastases. Based on our case and the literature that we reviewed, MiNENs represent an extremely rare diagnosis. Due to diagnostic limitations and insufficient scientific data, their prevalence might be largely underestimated. Preoperative diagnosis is difficult without specific symptoms and radiological features. Thus, the possibility of MiNEN should be considered when abnormal pancreatic lesions are detected.

\section{Acknowledgments}

Funding: This work was jointly supported by National Natural Science Foundation (No. 81972250, No. 81871950 and No. 81972725); the National Science Foundation for Distinguished Young Scholars of China (No. 81625016); Clinical and Scientific Innovation Project of Shanghai Hospital Development Center (SHDC12018109); Clinical Research Plan of Shanghai Hospital Development Center (SHDC2020CR1006A); Scientific Innovation Project of Shanghai Education Committee (2019-01-07-00-07E00057).

\section{Footnote}

Reporting Checklist: The authors have completed the CARE reporting checklist. Available at https://dx.doi. org/10.21037/gs-21-564

Conflicts of Interest: All authors have completed the ICMJE uniform disclosure form (available at https://dx.doi. org/10.21037/gs-21-564). The authors have no conflicts of interest to declare.

Ethical Statement: The authors are accountable for all aspects of the work in ensuring that questions related to the accuracy or integrity of any part of the work are appropriately investigated and resolved. All procedures performed in studies involving human participants were in accordance with the ethical standards of the institutional and/or national research committee(s) and with the Helsinki Declaration (as revised in 2013). Written informed consent was obtained from the patient for publication of this case report and accompanying images. A copy of the written consent is available for review by the editorial office of this journal.

Open Access Statement: This is an Open Access article distributed in accordance with the Creative Commons Attribution-NonCommercial-NoDerivs 4.0 International License (CC BY-NC-ND 4.0), which permits the noncommercial replication and distribution of the article with the strict proviso that no changes or edits are made and the original work is properly cited (including links to both the formal publication through the relevant DOI and the license). See: https://creativecommons.org/licenses/by-nc$\mathrm{nd} / 4.0 \%$.

\section{References}

1. Lewin K. Carcinoid tumors and the mixed (composite) glandular-endocrine cell carcinomas. Am J Surg Pathol 1987;11 Suppl 1:71-86.

2. Capella C, La Rosa S, Uccella S, et al. Mixed endocrineexocrine tumors of the gastrointestinal tract. Semin Diagn Pathol 2000;17:91-103.

3. Inzani F, Petrone G, Rindi G. The New World Health Organization Classification for Pancreatic Neuroendocrine Neoplasia. Endocrinol Metab Clin North Am 2018;47:463-70.

4. La Rosa S, Sessa F, Uccella S. Mixed NeuroendocrineNonneuroendocrine Neoplasms (MiNENs): Unifying the Concept of a Heterogeneous Group of Neoplasms. Endocr Pathol 2016;27:284-311.

5. Yang M, Ke NW, Zhang Y, et al. Survival Analyses for Patients With Surgically Resected Pancreatic Neuroendocrine Tumors by World Health Organization 2010 Grading Classifications and American Joint Committee on Cancer 2010 Staging Systems. Medicine (Baltimore) 2015;94:e2156.

6. Frizziero M, Wang X, Chakrabarty B, et al. Retrospective study on mixed neuroendocrine non-neuroendocrine neoplasms from five European centres. World J Gastroenterol 2019;25:5991-6005.

7. Carideo L, Prosperi D, Panzuto F, et al. Role of Combined [68Ga]Ga-DOTA-SST Analogues and [18F]FDG PET/ CT in the Management of GEP-NENs: A Systematic Review. J Clin Med 2019;8:1032.

8. Evangelista L, Ravelli I, Bignotto A, et al. Ga-68 DOTA-peptides and F-18 FDG PET/CT in patients 
with neuroendocrine tumor: A review. Clin Imaging 2020;67:113-6.

9. Frizziero M, Chakrabarty B, Nagy B, et al. Mixed Neuroendocrine Non-Neuroendocrine Neoplasms: A Systematic Review of a Controversial and Underestimated Diagnosis. J Clin Med 2020;9:273.

10. La Rosa S, Marando A, Sessa F, et al. Mixed Adenoneuroendocrine Carcinomas (MANECs) of the Gastrointestinal Tract: An Update. Cancers (Basel) 2012;4:11-30.

11. Nießen A, Schimmack S, Weber TF, et al. Presentation and outcome of mixed neuroendocrine non-neuroendocrine neoplasms of the pancreas. Pancreatology 2021;21:224-35.

12. Schiavo Lena M, Cangi MG, Pecciarini L, et al. Evidence of a common cell origin in a case of pancreatic mixed intraductal papillary mucinous neoplasm-neuroendocrine tumor. Virchows Arch 2021;478:1215-9.

13. Varshney B, Bharti JN, Varshney VK, et al. Mixed neuroendocrine-non-neuroendocrine neoplasms (MiNEN) of pancreas: a rare entity-worth to note. BMJ Case Rep 2020;13:234855.

14. Shimada N, Miwa S, Arai T, et al. Cystic mixed adenoneuroendocrine carcinoma of the pancreas: A case report. Int J Surg Case Rep 2018;52:1-4.

15. Li X, Li D, Sun X, et al. Mixed adenoneuroendocrine carcinoma (MANEC) of the ampulla of Vater in a Chinese patient: A case report. J Int Med Res 2020;48:300060520947918.

16. Mori H, Hanada K, Minami T, et al. A case of mixed adenoneuroendocrine carcinoma of the pancreas mimicking intraductal papillary mucinous carcinoma. Clin J Gastroenterol 2018;11:320-6.

17. Strait AM, Sharma N, Tsapakos MJ, et al. Pancreatic mixed acinar-neuroendocrine carcinoma, a unique diagnostic challenge on FNA cytology: A small series of two cases with literature review. Diagn Cytopathol 2018;46:971-6.

18. Kaji K, Seishima J, Yamato M, et al. Clinical utility of endoscopic ultrasound-guided fine-needle aspiration in mixed adenoneuroendocrine carcinoma with signetring cells of the pancreas: a case report and review of the literature. Clin J Gastroenterol 2016;9:43-8.

19. Zhang L, DeMay RM. Cytological features of mixed adenoneuroendocrine carcinoma of the ampulla: two case reports with review of literature. Diagn Cytopathol 2014;42:1075-84.
20. Ogbonna OH, Garcon MC, Syrigos KN, et al. Mixed acinar-neuroendocrine carcinoma of the pancreas with neuroendocrine predominance. Case Rep Med 2013;2013:705092.

21. Lee L, Bajor-Dattilo EB, Das K. Metastatic mixed acinarneuroendocrine carcinoma of the pancreas to the liver: a cytopathology case report with review of the literature. Diagn Cytopathol 2013;41:164-70.

22. Imaoka K, Fukuda S, Tazawa H, et al. A mixed adenoneuroendocrine carcinoma of the pancreas: a case report. Surg Case Rep 2016;2:133.

23. Huang Z, Xiao WD, Li Y, et al. Mixed adenoneuroendocrine carcinoma of the ampulla: two case reports. World J Gastroenterol 2015;21:2254-9.

24. Gao G, Qorbani A, Zhou CH. A pancreatic mixed neuroendocrine-non-neuroendocrine neoplasms (MiNEN) (NET and undifferentiated carcinoma of the pancreas with osteoclast-like giant cells) with metastatic neuroendocrine component to the liver. Autops Case Rep 2020;11:e2020201.

25. Murata M, Takahashi H, Yamada M, et al. A case of mixed adenoneuroendocrine carcinoma of the pancreas: Immunohistochemical analysis for histogenesis. Medicine (Baltimore) 2017;96:e6225.

26. Leteurtre E, Brami F, Kerr-Conte J, et al. Mixed ductal-endocrine carcinoma of the pancreas: a possible pathogenic mechanism for arrhythmogenic right ventricular cardiomyopathy. Arch Pathol Lab Med 2000;124:284-6.

27. Chatelain D, Parc Y, Christin-Maitre S, et al. Mixed ductal-pancreatic polypeptide-cell carcinoma of the pancreas. Histopathology 2002;41:122-6.

28. Scardoni M, Vittoria E, Volante M, et al. Mixed adenoneuroendocrine carcinomas of the gastrointestinal tract: targeted next-generation sequencing suggests a monoclonal origin of the two components. Neuroendocrinology 2014;100:310-6.

29. Uccella S, La Rosa S. Looking into digestive mixed neuroendocrine - nonneuroendocrine neoplasms: subtypes, prognosis, and predictive factors. Histopathology 2020;77:700-17.

30. Pokrzywa CJ, Abbott DE, Matkowskyj KA, et al. Natural History and Treatment Trends in Pancreatic Cancer Subtypes. J Gastrointest Surg 2019;23:768-78.

31. Düzköylü Y, Aras O, Bostancı EB, et al. Mixed AdenoNeuroendocrine Carcinoma; Case Series of Ten Patients with Review of the Literature Balkan Med J 2018;35:263-7. 
32. Kim JY, Brosnan-Cashman JA, Kim J, et al. Pancreatic acinar cell carcinomas and mixed acinar-neuroendocrine carcinomas are more clinically aggressive than grade

Cite this article as: Wang F, Lou X, Qin Y, Xu X, Yu X, Huang D, Ji S. Mixed neuroendocrine nonneuroendocrine neoplasms of the pancreas: a case report and literature review of pancreatic mixed neuroendocrine nonneuroendocrine neoplasm. Gland Surg 2021;10(12):3443-3452. doi: 10.21037/gs21-564
1 pancreatic neuroendocrine tumours. Pathology 2020;52:336-47. 
Supplementary

\section{Appendix}

PubMed:

(pancrea*[Title/Abstract]) AND ((((Mixed neuroendocrine-non neuroendocrine neoplasms [Title/Abstract]) OR (MiNEN[Title/Abstract])) OR (mixed adeno-neuroendocrine carcinoma[Title/Abstract])) OR (MANEC[Title/Abstract]))

Web of Science:

$(((T S=($ Mixed neuroendocrine-non neuroendocrine neoplasms $))$ OR TS= $($ MiNEN $))$ OR TS $=($ mixed adenoneuroendocrine carcinoma)) OR TS=(MANEC) 